\title{
Gamow-Teller strength distributions in Xe isotopes
}

\author{
O. Moreno, ${ }^{1}$ R. Álvarez-Rodríguez, ${ }^{1}$ P. Sarriguren, ${ }^{1}$ E. Moya de Guerra, ${ }^{1,2}$ J. M. Udías, ${ }^{2}$ and J. R. Vignote ${ }^{1,2}$ \\ ${ }^{1}$ Instituto de Estructura de la Materia. Consejo Superior de Investigaciones Científicas. Serrano 123, E-28006 Madrid, Spain \\ ${ }^{2}$ Departamento de Física Atómica, Molecular y Nuclear. Facultad de Ciencias Físicas. Universidad Complutense de Madrid. \\ Avenida Complutense s/n, E-28040 Madrid, Spain
}

(Received 5 June 2006; published 16 November 2006)

\begin{abstract}
The energy distributions of the Gamow-Teller (GT) strength are studied for even-even Xe isotopes with mass numbers from 124 to 142 . A self-consistent microscopic formalism is used to generate the single-particle basis, using a deformed Skyrme Hartree-Fock mean field with pairing correlations in the BCS approximation. The GT transitions are obtained within a quasiparticle random phase approximation approach using a residual spin-isospin interaction in the particle-hole (ph) and particle-particle (pp) channels. We then discuss the pairing BCS treatment and the determination of the ph and pp residual interaction coupling constants. We study the $\mathrm{GT}^{+}$and $\mathrm{GT}^{-}$strength distributions for the equilibrium nuclear shapes, which comprise essential information for studies of charge-exchange reactions and double- $\beta$ processes involving these isotopes.
\end{abstract}

DOI: 10.1103/PhysRevC.74.054308

PACS number(s): 21.60.Jz, 23.40.-s, 24.30.Gd, 27.60.+j

\section{INTRODUCTION}

The isotope ${ }^{136} \mathrm{Xe}$ has been recently used as a moving target in collisions with a hydrogen gas jet in a first test to prove the feasibility of the EXL experimental approach at FAIR-GSI [1]. Charge-exchange $(p, n)$ reactions on this $\mathrm{Xe}$ isotope were consequently measured. Although this kind of facility is intended to explore highly unstable nuclei, some stable isotopes such as those under study here are normally used as initial test targets. Therefore, having reliable information on their nuclear structure turns out to be of primary importance. From ${ }^{136} \mathrm{Xe}$, we extend our study to other stable isotopes, ${ }^{124-134} \mathrm{Xe}$, and also to the neutron-rich region ${ }^{138,140,142} \mathrm{Xe}$. The latter undergo $\beta^{-}$decay and their half-lives provide us with another piece of experimental information.

Gamow-Teller transition matrix elements can be extracted from the measured forward-angle charge-exchange data [2]. At high incident energies and at forward angles, the nuclear states are probed at small momentum transfer. Therefore, only the central parts of the isovector effective interaction contribute to the cross section. Furthermore, because of the small momentum transfer, a multipole expansion leads to a simple relation between the measured $0^{\circ}$ cross sections and the corresponding allowed $\beta$-decay transition rates $(L=0)$. Taking into account the projectile distortion effects in the distorted-wave Born approximation (DWBA), the transition amplitudes still approximately factorize into a nuclear reaction part and a nuclear structure part. Therefore, the $0^{\circ}$ charge-exchange cross sections are still proportional to the corresponding Fermi and Gamow-Teller matrix elements. The proportionality factor is parametrized in terms of a distortion factor, a kinematic factor, and a volume integral of the effective interaction [2].

Theoretical $\mathrm{GT}^{ \pm}$strength distributions such as the ones discussed here can therefore be used to predict cross sections of various charge-exchange reactions under the appropriate kinematic conditions. Some of the charge-exchange reactions corresponding to $\mathrm{GT}^{-}$processes are as follows:

${ }^{136} \mathrm{Xe}(p, n){ }^{136} \mathrm{Cs} ; \quad{ }^{136} \mathrm{Xe}\left({ }^{3} \mathrm{He},{ }^{3} \mathrm{H}\right){ }^{136} \mathrm{Cs} ; \quad{ }^{136} \mathrm{Xe}(d, 2 n){ }^{136} \mathrm{Cs}$.

Some examples of those corresponding to $\mathrm{GT}^{+}$transitions are

$$
{ }^{136} \mathrm{Xe}(n, p){ }^{136} \mathrm{I} ; \quad{ }^{136} \mathrm{Xe}\left({ }^{3} \mathrm{H},{ }^{3} \mathrm{He}\right){ }^{136} \mathrm{I} ; \quad{ }^{136} \mathrm{Xe}(d, 2 p){ }^{136} \mathrm{I} .
$$

In addition, the $\mathrm{Xe}$ isotopes are of special interest for double- $\beta$ processes [3]. Different nuclear models have been developed by many groups to calculate quantitatively the double- $\beta$ matrix elements, as described in recent review articles [4]. Table I shows all the transitions of this type involving $\mathrm{Xe}$ isotopes as parents or daughters. The $\mathrm{GT}^{ \pm}$ strength distributions that will be obtained here can be used to calculate the transition amplitudes of the initial and final ground states going to the virtual quasiparticle random phase approximation (QRPA) excited states of the intermediate odd-odd nucleus. After the overlap between the intermediate states coming from the parent and from the daughter nuclei is computed, the two-neutrino double- $\beta$ decay matrix elements as well as the half-lives can be calculated. Such a calculation has indeed been carried out in [8] for ${ }^{128} \mathrm{Xe},{ }^{130} \mathrm{Xe}$, and ${ }^{136} \mathrm{Xe}$, which are $\beta^{-} / \beta^{-}$parents or daughters.

Finally, Xe isotopes are of considerable interest because they belong to a typical shape transitional region [9], in which there are experimental indications of triaxial deformation in some isotopes [10]. Present theoretical triaxial calculations are mainly based on algebraic models [11]. Nevertheless, in this work we assume axial symmetry. As we shall see, for the deformed $\mathrm{Xe}$ isotopes with two equilibrium shapes there are no critical changes in the Gamow-Teller strengths at these two shapes, and a similar behavior may be expected when considering possible triaxial shapes. In any case, axial deformation is a crucial ingredient of the formalism that gives rise to new features in the Gamow-Teller strength distributions that are different from those obtained within a spherical treatment.

Although some of the isotopes studied may be spherical, in this work we are not restricted to those but we deal with 
TABLE I. Double- $\beta$ processes involving Xe isotopes, with their experimental half-lives [5,6] and $Q$ values from experimental masses [7]. When a $\beta^{+} / \beta^{+}$transition is indicated, $\beta^{+} / \mathrm{EC}$ and $\mathrm{EC} / \mathrm{EC}$ are also allowed (with $Q$ values 1.022 and $2.044 \mathrm{MeV}$ lower than the one shown, respectively).

\begin{tabular}{lccc}
\hline \hline Transition & $2 \beta$ process & $T_{1 / 2}(\mathrm{yr})$ exp. & $Q$ value $(\mathrm{MeV})$ \\
\hline${ }^{\mathbf{1 2 4}} \mathbf{X e} \rightarrow{ }^{124} \mathrm{Te}$ & $\beta^{+} / \beta^{+}$ & $>2.0 \times 10^{14}[5]$ & 2.866 \\
${ }^{\mathbf{1 2 6}} \mathbf{X e} \rightarrow{ }^{126} \mathrm{Te}$ & $\mathrm{EC} / \mathrm{EC}$ & - & 0.897 \\
${ }^{128} \mathrm{Te} \rightarrow{ }^{\mathbf{1 2 8}} \mathbf{X e}$ & $\beta^{-} / \beta^{-}$ & $2.5 \pm 0.3 \times 10^{24}[6]$ & 0.867 \\
${ }^{130} \mathrm{Te} \rightarrow{ }^{\mathbf{1 3 0}} \mathbf{X e}$ & $\beta^{-} / \beta^{-}$ & $0.9 \pm 0.1 \times 10^{21}[6]$ & 2.529 \\
${ }^{130} \mathrm{Ba} \rightarrow{ }^{\mathbf{1 3 0}} \mathbf{X e}$ & $\beta^{+} / \beta^{+}$ & $>4.0 \times 10^{21}[5]$ & 2.610 \\
${ }^{132} \mathrm{Ba} \rightarrow{ }^{\mathbf{1 3 2}} \mathbf{X e}$ & $\mathrm{EC} / \mathrm{EC}$ & $>3.0 \times 10^{20}[5]$ & 0.840 \\
${ }^{{ }^{134} \mathbf{X e} \rightarrow{ }^{134} \mathrm{Ba}}$ & $\beta^{-} / \beta^{-}$ & $>1.1 \times 10^{16}[5]$ & 0.830 \\
${ }^{136} \mathbf{X e} \rightarrow{ }^{136} \mathrm{Ba}$ & $\beta^{-} / \beta^{-}$ & $8.1 \times 10^{20}[5]$ & 2.468 \\
\hline \hline
\end{tabular}

a large number of $\mathrm{Xe}$ isotopes whose equilibrium shapes are unknown. For most of the isotopes considered here, there is no clear experimental evidence of whether they are spherical or deformed. Under these circumstances, a deformed approach is always preferable over a spherical one because a deformed formalism contains the spherical shape as a particular solution.

We have also found in the past $[12,13]$ that the GT strength distributions may depend, in some cases significantly, on the deformation of the decaying nucleus. But we notice that this dependence has to be studied case by case since it is very sensitive to the fragmentation and crossing of levels generated by the deformation. We think it is indeed worth studying the degree of sensitivity of the GT strength of these Xe isotopes to deformation.

Following this introduction we present in Sec. II a brief description of the theoretical framework. Section III includes our results regarding $\mathrm{HF}+\mathrm{BCS}$ energies and $\mathrm{GT}^{ \pm}$strength distributions, together with a discussion on the pairing treatment. Finally, Sec. IV contains the main conclusions of our work.

\section{THEORETICAL FRAMEWORK}

We describe here briefly the theoretical formalism used, the details of which can be found in Ref. [12]. We carry out a deformed Hartree-Fock calculation with the effective nucleon-nucleon density-dependent Skyrme interaction Sk3 [14], assuming axial deformation and time-reversal symmetry. The single-particle wave functions are expanded in terms of the eigenstates of an axially symmetric harmonic oscillator in cylindrical coordinates using eleven major shells. Pairing correlations between like nucleons are included in the BCS approximation by taking either fixed pairing gap parameters ( $\Delta_{\pi}$ for protons and $\Delta_{\nu}$ for neutrons) or fixed pairing strength parameters $\left(G_{\pi}\right.$ and $G_{\nu}$, respectively). We refer to these two types of calculations as $\operatorname{HF}(\operatorname{Sk} 3)+\operatorname{BCS}(\Delta)$ and $\mathrm{HF}(\mathrm{Sk} 3)+\mathrm{BCS}(G)$, respectively. They yield single-particle energies and wave functions together with their occupation probabilities for protons and neutrons separately. A quadrupole-constrained HF + BCS calculation [15], where the intrinsic quadrupole moment is constrained, is also performed to obtain the deformation dependence of the ground-state energy.
The pairing energy gaps $\Delta$ are determined phenomenologically, and the pairing strengths $G$ are obtained from them in an indirect way, as will be described later. Within the BCS approximation, both parameters are related by the so-called gap equation:

$$
\Delta=G \sum_{i} u_{i} v_{i}
$$

where $v_{i}$ and $u_{i}$ are occupation and nonoccupation probability amplitudes of the $i$ th single-particle level subject to the condition $v_{i}^{2}+u_{i}^{2}=1$. To determine the value of $G$ that reproduces a given value of $\Delta$, one should take into account that this depends on the active energy range and number of levels considered. In our case, we include all HF single-particle sates in our basis above and below the Fermi level. It is important to stress that the occupation probability amplitudes are computed in each iteration of the HF method, and these are used to calculate the one-body density and mean field of the next iteration, so that one gets new single-particle wave functions, energies, and occupation numbers at each iteration. Therefore, the self-consistent determination of the binding energy and deformation includes pairing correlations from the beginning.

To describe Gamow-Teller excitations we add to the quasiparticle mean field a separable spin-isospin residual interaction in the particle-hole (ph) and particle-particle (pp) channels, which is treated within the QRPA. The advantage of using separable forces is that the QRPA energy eigenvalue problem is reduced to finding the roots of an algebraic equation. The ph part,

$V_{\mathrm{GT}}^{\mathrm{ph}}=2 \chi_{\mathrm{GT}}^{\mathrm{ph}} \sum_{K=0, \pm 1}(-1)^{K} \beta_{K}^{+} \beta_{-K}^{-}, \quad \beta_{K}^{+}=\sum_{\pi \nu}\left\langle\nu\left|\sigma_{K}\right| \pi\right\rangle a_{\nu}^{+} a_{\pi}$,

is responsible for the position and structure of the GT resonance [12,16-18]. The corresponding coupling constant $\chi_{\mathrm{ph}}^{\mathrm{GT}}$ is obtained in a consistent way from the same energy density functional as the Hartree-Fock mean field through a second derivative with respect to the nucleonic density and by averaging the contact interaction over the nuclear volume [12]. The pp part consists of a proton-neutron pairing force, which we introduce as a separable force $[17,19,20]$,

$$
\begin{aligned}
V_{\mathrm{GT}}^{\mathrm{pp}} & =-2 \kappa_{\mathrm{GT}}^{\mathrm{pp}} \sum_{K}(-1)^{K} P_{K}^{+} P_{-K}, \\
P_{K}^{+} & =\sum_{\pi v}\left\langle\pi\left|\left(\sigma_{K}\right)^{+}\right| \nu\right\rangle a_{\nu}^{+} a_{\bar{\pi}}^{+} .
\end{aligned}
$$

The coupling constant $\kappa_{\mathrm{pp}}^{\mathrm{GT}}$ may in principle be derived consistently with the HFB or $\mathrm{HF}+\mathrm{BCS}$ mean field through a second derivative with respect to the pairing tensor of the energy density functional. This derivation would be analogous to the way in which the ph force is obtained as the second derivative with respect to the density. In our theoretical scheme the proton-neutron pairing interaction is neglected in the construction of the mean field to avoid mixing of even-even and odd-odd isotopes in the intrinsic state. Only pairing between like particles is included in the BCS approximation. This implies that the particle-particle interaction in the protonneutron channel is in principle undetermined. Therefore the 
coupling constant $\kappa_{\mathrm{pp}}^{\mathrm{GT}}$ is fitted to the phenomenology, as for example to reproduce half-lives as is usually done $[17,18]$.

The $p n$ QRPA phonon operator for GT excitations in eveneven nuclei is written as

$$
\Gamma_{\omega_{K}}^{+}=\sum_{\pi \nu}\left[X_{\pi \nu}^{\omega_{K}} \alpha_{\nu}^{+} \alpha_{\bar{\pi}}^{+}-Y_{\pi \nu}^{\omega_{K}} \alpha_{\bar{\nu}} \alpha_{\pi}\right]
$$

where $\pi$ and $v$ stand for proton and neutron, respectively, $\alpha^{+}(\alpha)$ are quasiparticle creation (annihilation) operators, $\omega_{K}$ are the RPA excitation energies, and $X_{\pi \nu}^{\omega_{K}}$ and $Y_{\pi \nu}^{\omega_{K}}$ are the forward and backward amplitudes, respectively. The operator satisfies

$$
\Gamma_{\omega_{K}}|0\rangle=0 ; \quad \Gamma_{\omega_{K}}^{+}|0\rangle=\left|\omega_{K}\right\rangle,
$$

when acting on the QRPA ground state of the parent nucleus, $|0\rangle$.

The technical details for solving the QRPA equations are well described in Refs. [12,16,19]. Here we only mention that, because of the use of separable residual forces, the solutions of the QRPA equations are found by first solving a dispersion relation, which is an algebraic equation of fourth order in the excitation energy $\omega$. Then, for each value of the energy, the GT transition amplitudes in the intrinsic frame connecting the ground state $|0\rangle$ to one-phonon states in the daughter nucleus, $\left|\omega_{K}\right\rangle$, are determined by using the normalization conditions of the phonon amplitudes. They are given by

$$
\left\langle\omega_{K}\left|\beta_{K}^{ \pm}\right| 0\right\rangle=\mp M_{ \pm}^{\omega_{K}},
$$

where

$$
\begin{aligned}
& M_{-}^{\omega_{K}}=\sum_{\pi v}\left(q_{\pi v} X_{\pi v}^{\omega_{K}}+\tilde{q}_{\pi v} Y_{\pi v}^{\omega_{K}}\right) \\
& M_{+}^{\omega_{K}}=\sum_{\pi v}\left(\tilde{q}_{\pi v} X_{\pi v}^{\omega_{K}}+q_{\pi v} Y_{\pi v}^{\omega_{K}}\right),
\end{aligned}
$$

with

$\tilde{q}_{\pi v}=u_{v} v_{\pi} \Sigma_{K}^{v \pi} ; \quad q_{\pi v}=v_{v} u_{\pi} \Sigma_{K}^{v \pi} ; \quad \Sigma_{K}^{v \pi}=\left\langle v\left|\sigma_{K}\right| \pi\right\rangle$.

It is a simple matter to verify that the Ikeda sum rule

$$
\sum_{\omega_{K}}\left[\left(M_{-}^{\omega_{K}}\right)^{2}-\left(M_{+}^{\omega_{K}}\right)^{2}\right]=3(N-Z)
$$

holds in the RPA approximation, provided all the eigenvalues contained in the basis space are included in the sum, so that the orthonormalization conditions are satisfied. In practice, the strength functions are calculated up to an energy $\omega<E_{\text {cut }}$, where $E_{\text {cut }}$ is such that the Ikeda sum rule is fulfilled up to a few per thousand discrepancy. Typical energies used in our calculations are $E_{\text {cut }}=30 \mathrm{MeV}$. The number of configurations involved in this mass region for this energy range is typically over 1000.

Once the intrinsic amplitudes in Eq. (2.6) are calculated, the Gamow-Teller strength $B(\mathrm{GT})$ in the laboratory frame for a transition $I_{i} K_{i}\left(0^{+} 0\right) \rightarrow I_{f} K_{f}\left(1^{+} K\right)$ can be obtained as

$$
\begin{aligned}
B^{ \pm}(\mathrm{GT})= & \sum_{M_{i}, M_{f}, \mu}\left|\left\langle I_{f} M_{f}\left|\beta_{\mu}^{ \pm}\right| I_{i} M_{i}\right\rangle\right|^{2} \\
= & \left\{\delta_{K_{f}, 0}\left\langle\phi_{K_{f}}\left|\beta_{0}^{ \pm}\right| \phi_{0}\right\rangle^{2}\right. \\
& \left.+2 \delta_{K_{f}, 1}\left\langle\phi_{K_{f}}\left|\beta_{1}^{ \pm}\right| \phi_{0}\right\rangle^{2}\right\} .
\end{aligned}
$$

To obtain this expression we have used the initial and final states in the laboratory frame expressed in terms of the intrinsic states $\left|\phi_{K}\right\rangle$, using the Bohr and Mottelson factorization [21]. Theoretical $\beta^{-}$-decay half-lives are calculated by summing all the energetically allowed transition probabilities in (2.10), in units of $g_{A}^{2} / 4 \pi$, weighted with phase-space factors, up to states in the daughter nucleus with excitation energies below the $Q_{\beta^{-}}$window.

One may wonder whether, in deformed nuclei, the calculated GT strengths may contain spurious contributions from higher angular momentum components in the initial and final wave functions. As previously mentioned, the GT strengths are calculated in the laboratory frame in the factorization approximation of Bohr and Mottelson. By using angular momentum projection techniques [22], the angular momentum projection can be carried out through an expansion in inverse powers of the angular momentum operator component perpendicular to the symmetry axis, $\left\langle J_{\perp}^{2}\right\rangle$. This expansion, to lower order, provides a factorization approximation formally identical to that of Bohr and Mottelson. Thus, the effect of angular momentum projection is to a large extent taken into account. An upper bound to contributions from higher angular momentum components is proportional to $\left\langle J_{\perp}^{2}\right\rangle^{-2}$, with values of $\left\langle J_{\perp}^{2}\right\rangle$ ranging from 10 to 40 in the case of the deformed $\mathrm{Xe}$ isotopes. Therefore, exact angular momentum projection in deformed Xe isotopes would lead in all cases to less than a few percent effect in the GT strengths. In the cases where the shape is spherical, there are no high angular momentum contributions to the GT strengths.

It may be questioned whether it is correct to introduce additional BCS parameters and residual interactions on top of the Skyrme interaction, which is already an effective interaction. Indeed, if the effective force used in constructing the mean field were to be the most general possible interaction, one should not include additional parameters. However, this is not the case for the Skyrme interaction. The parameters of the Skyrme force are determined by requiring that they reproduce the nuclear compressibility, as well as the total binding energies and charge radii of magic nuclei in spherical self-consistent calculations. It is well known that the effective Skyrme interaction and its existent parametrizations are suitable for generating the optimal HF mean field of spherical and deformed nuclei [14,23]. The particle and hole eigenstates of the mean field, which form the canonical basis, have highly nontrivial wave functions that contain a mixture of many harmonic oscillator shells, when expanded in a harmonic oscillator basis. In addition to deformation, for non-closed-shell nuclei, one has to take into account pairing correlation effects, which are important when the level density around the Fermi level becomes large.

It is also well known that the effective Skyrme interaction is unsuitable for generating the quasiparticle mean field, since in the fit of the Skyrme forces no attention is paid to the realistic character of the pairing matrix elements, and extensions of the Skyrme HF method have been developed over the years. To include pairing correlations in the mean field one possibility is to do Hartree-Fock-Bogoliubov calculations using either finite-range forces, such as the Gogny force, or contact densitydependent pairing interactions [24]. All of these forces are 
extensions of the Skyrme forces specifically designed for this purpose. The other possibility is to perform BCS calculations in the canonical basis using either phenomenological fixed-gap parameters or fixed pairing strengths, as originally proposed by Vautherin. This is the path followed in our paper. This path has proven to be successful for studying the properties of the ground state and low-spin excited states in open-shell nuclei $[12,14,23,25]$.

As already mentioned, the same effective Skyrme interaction is used to generate a separable particle-hole residual interaction. The separable interaction simplifies enormously the calculation and still contains the main characteristics of the contact force. The quasiparticle energy density functional obtained with the effective Skyrme and pairing interactions that we used does not contain any dependence on particleparticle interactions in the proton-neutron channel. Therefore we have to introduce a proton-neutron particle-particle residual interaction in the usual way as a separable force with a coupling strength that we fit to the measured half-lives. Recall that a bridge between Skyrme HF and RPA calculations for excited states was established long ago [26] by using a particle-hole force in the RPA, which is determined by the second derivatives of the HF energy with respect to the density. But there is no guarantee that the derived force is good for excited states. This is so because Skyrme forces are constructed for the description of ground-state properties. Finally, notice that similar schemes based on Skyrme HF + BCS + RPA have been frequently used in the literature with successful results [27].

\section{RESULTS AND DISCUSSION}

\section{A. Equilibrium deformations}

A deformed Hartree-Fock mean-field calculation is performed using a Sk3 Skyrme force with a constraint of the quadrupole deformation given by the parameter $\beta=$ $\sqrt{\pi / 5} Q_{p} /\left(Z\left\langle r^{2}\right\rangle\right)$, where $Q_{p}$ is the proton quadrupole moment and $\left\langle r^{2}\right\rangle$ is the charge mean-square radius. Also included is a pairing interaction between like nucleons within the BCS approximation, in which the pairing energy gap $(\Delta)$ or the pairing strength $(G)$ is kept fixed. From this calculation we obtain the ground-state energy as a function of the quadrupole deformation $\beta$.

In principle, when a pairing force with fixed strength $G_{\pi, v}$ is used, the pairing energy gaps $\Delta_{\pi, \nu}$ depend on the strength of the pairing interaction as well as on the occupation amplitudes of the single-particle states [see Eq. (2.1)]. This last condition amounts to saying that pairing energy gaps are deformation dependent. Actually, we obtain these gaps first phenomenologically from the odd-even mass differences by means of a symmetric five-term formula involving experimental binding energies [7], and we keep them fixed to carry out a deformation-constrained $\mathrm{HF}+\mathrm{BCS}(\Delta)$ calculation. Next we fix the pairing strength so as to reproduce the gap parameters at the deformation of the ground state, and we carry out a deformation-constrained $\mathrm{HF}+\mathrm{BCS}(G)$ calculation. In this way we perform a fixed-pairing-strength calculation, which is conceptually more appealing, but still profit from the experimental information available for the gaps.
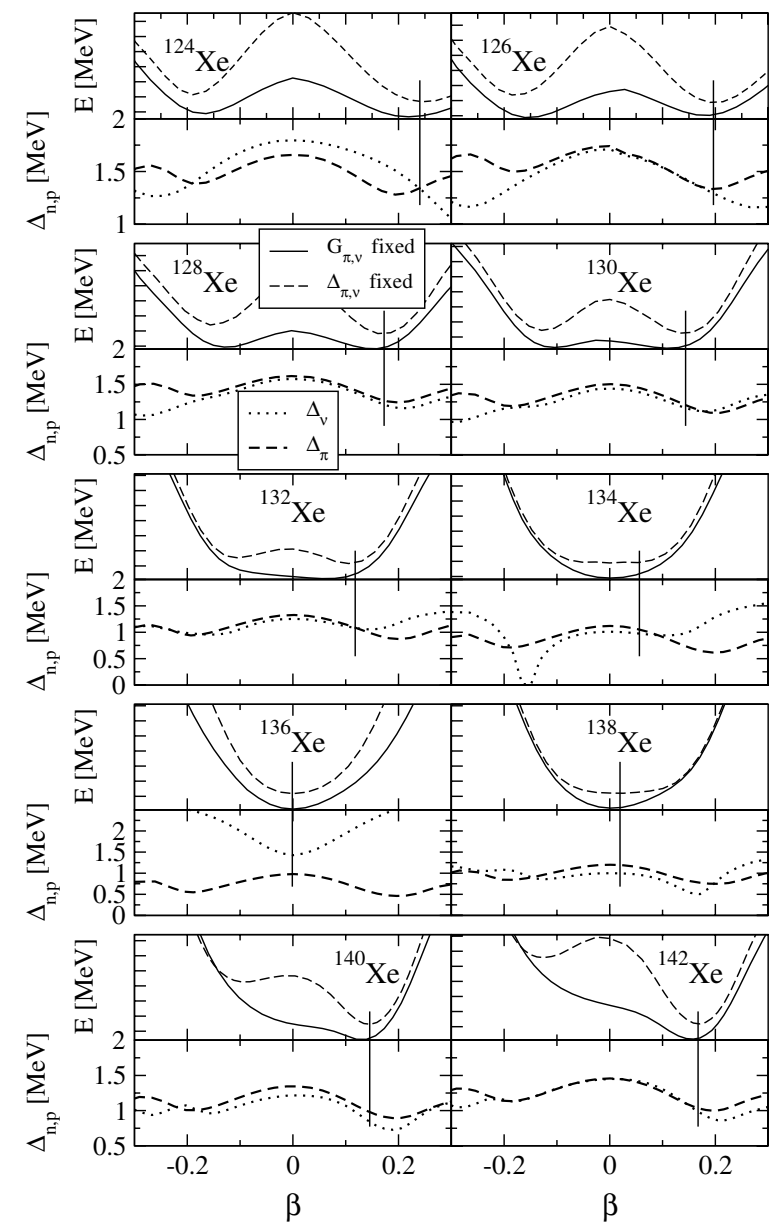

FIG. 1. HF(Sk3) + BCS energy of the isotopes ${ }^{124-142} \mathrm{Xe}$ as a function of the quadrupole deformation $\beta$ for fixed pairing gap (dashed line) and for fixed pairing strength (solid line) treatments (with $1 \mathrm{MeV}$ of separation between absolute minima), as well as deformation dependence of pairing gaps from the fixed strength calculation (dashed line for proton gap and dotted line for neutron gap). The scale in the vertical axis is $1 \mathrm{MeV}$ between ticks. Vertical lines indicate ground states from the fixed-gap calculation.

Figure 1 shows the HF + BCS energies for ${ }^{124-142} \mathrm{Xe}$, using the Sk3 Skyrme interaction. The pairing interaction is included in both fixed-gap (dashed line) and fixed-strength (solid line) treatments, each of them in a different curve whose absolute minima have been separated $1 \mathrm{MeV}$ for a better comparison. For the same isotope, both curves show energy minima at very similar deformations, with the ones corresponding to the fixed pairing strength treatment being slightly smaller in absolute value. One can also observe that the energy barrier at the spherical region of ${ }^{124-132} \mathrm{Xe}$ and of ${ }^{140-142} \mathrm{Xe}$ is less pronounced when the pairing strength is fixed, and in this case both minima have very similar energy (with the prolate one being generally the ground state, except for ${ }^{126} \mathrm{Xe}$ ). As the mass number increases from $A=124$ to $A=138$, the deformations of the equilibrium shapes decrease and eventually converge to a spherical shape. The two final isotopes show only one equilibrium deformation in the prolate region. 
Underneath each of these energy-deformation graphs we plot the corresponding pairing gaps as a function of the quadrupole deformation for the fixed-pairing-strength calculation. The vertical lines join the ground states from the fixed-gap treatment with the corresponding pairing gaps at this deformation from the fixed-strength treatment. These values are those coming from the aforementioned odd-even experimental mass differences.

In our calculations with fixed $G$ values the binding energies show a tendency to increase at those deformations where the pairing gaps reach a minimum. This appears contradictory since one may expect the opposite because the smaller the pairing gap is, the lower is the contribution of the pairing energy to the total binding energy. However, it indicates that the minima of the pairing gaps appear at similar deformation to that where the volume and the spin-orbit term contributions to the binding energy are maximum. However, comparing calculations with $G$ fixed to those with $\Delta$ fixed, one sees in Fig. 1 that for $\beta$ values where $\Delta$ takes larger values the binding energy increases more compared to that obtained with lower ( $\Delta$ fixed) value.

In Table II we show the quadrupole deformation $\beta$ of the equilibrium shapes according to the $\operatorname{HF}(\operatorname{Sk} 3)+\operatorname{BCS}(\Delta)$ and $\operatorname{HF}(\operatorname{Sk} 3)+\operatorname{BCS}(G)$ calculations. We also show for comparison the results from independent theoretical calculations obtained from self-consistent relativistic calculations [28] as well as from phenomenological nonrelativistic calculations [29]. These results also indicate the existence of deformed solutions, which agree with those obtained here. Upper limits of the ground-state deformation obtained from experimental $B\left(E, 2_{1}^{+} \rightarrow 0_{1}^{+}\right)$transitions are also included [30]. Table III shows the pairing gaps, obtained from experimental binding energies, and the pairing strengths reproducing these gaps at the ground-state deformation.

\section{B. Gamow-Teller strength distributions}

The spin-isospin residual interactions in the particle-hole and particle-particle channels are treated here within a QPRA.
TABLE III. Pairing parameters for the HF + BCS calculation in ${ }^{124-142}$ Xe. Pairing gaps $\Delta_{v, \pi}$ are obtained from experimental binding energies [7]. The pairing strengths $G_{v, \pi}$ are those reproducing these gaps at the ground-state (gs) deformation, which is also indicated (see text for details).

\begin{tabular}{lccccc}
\hline \hline$A$ & $\Delta_{v}(\mathrm{MeV})$ & $\Delta_{\pi}(\mathrm{MeV})$ & $\beta_{\text {th. }} \mathrm{gs}$ & $G_{v}(\mathrm{MeV})$ & $G_{\pi}(\mathrm{MeV})$ \\
\hline 124 & 1.32 & 1.35 & 0.24 & 0.114 & 0.132 \\
126 & 1.31 & 1.33 & 0.19 & 0.111 & 0.133 \\
128 & 1.27 & 1.32 & 0.16 & 0.108 & 0.130 \\
130 & 1.25 & 1.31 & 0.13 & 0.108 & 0.129 \\
132 & 1.18 & 1.24 & 0.11 & 0.107 & 0.125 \\
134 & 1.01 & 1.12 & 0.05 & 0.107 & 0.119 \\
136 & 1.44 & 0.98 & 0.00 & 0.121 & 0.112 \\
138 & 1.00 & 1.20 & 0.03 & 0.098 & 0.120 \\
140 & 0.96 & 1.06 & 0.15 & 0.086 & 0.144 \\
142 & 1.03 & 1.06 & 0.17 & 0.084 & 0.116 \\
\hline \hline
\end{tabular}

The particle-hole residual interaction coupling constant $\chi_{\mathrm{ph}}$ is obtained consistently with the Hartree-Fock mean field, and their values vary from $0.21 \mathrm{MeV}$ in ${ }^{124} \mathrm{Xe}$ to $0.19 \mathrm{MeV}$ in ${ }^{142} \mathrm{Xe}$. We have used an average value for all the isotopes under study, $\chi_{\mathrm{ph}}=0.2 \mathrm{MeV}$. In the case of the particle-particle residual interaction, we have chosen the value of the coupling constant $\kappa_{\mathrm{pp}}$ so as to reproduce the experimental half-lives of the three unstable Xe isotopes: ${ }^{138} \mathrm{Xe}\left(T_{1 / 2}=844.8 \mathrm{~s}\right),{ }^{140} \mathrm{Xe}$ $\left(T_{1 / 2}=13.60 \mathrm{~s}\right)$, and ${ }^{142} \mathrm{Xe}\left(T_{1 / 2}=1.22 \mathrm{~s}\right)$. A good agreement between calculated and experimental $\beta^{-}$half-lives for the three isotopes is reached with $\kappa_{\mathrm{pp}}=0.07 \mathrm{MeV}$, provided we use the standard attenuation factor 0.77 for spin matrix elements as in previous works.

The single-particle energies and occupation probabilities at the equilibrium nuclear shapes are obtained from a $\operatorname{HF}(\operatorname{Sk} 3)+\operatorname{BCS}(G)$ calculation. Figure 2 shows $\mathrm{GT}^{-}$ strengths [in units of $g_{A}^{2} /(4 \pi)$ ] as a function of the excitation energy of the daughter nucleus after the transition. Discrete and Gaussian-folded distributions are shown; the latter are more suited to compare with experimental results regarding the GT strengths themselves or the cross sections of charge-exchange

TABLE II. Quadrupole deformation $\beta$ of the ${ }^{124-142}$ Xe equilibrium shapes from a HF(Sk3) + BCS calculation obtained with fixed pairing gaps $\Delta$ as well as with fixed pairing strengths $G$. Results from Refs. [28] and [29] are also given for comparison. Also given are the experimental values obtained from $B(E 2)$ transitions [30].

\begin{tabular}{|c|c|c|c|c|c|c|c|}
\hline \multirow[t]{2}{*}{$A$} & \multicolumn{2}{|c|}{$\beta_{\text {th. }}(\Delta$ fixed $)$} & \multicolumn{2}{|c|}{$\beta_{\text {th. }}(G$ fixed $)$} & \multirow[t]{2}{*}{$\beta_{\text {th. }}[28]$} & \multirow[t]{2}{*}{$\beta_{\text {th. }}[29]$} & \multirow{2}{*}{$\begin{array}{c}\left|\beta_{\text {exp. }}\right| \\
\text { from } B\left(E, 2_{1}^{+} \rightarrow 0_{1}^{+}\right)[30]\end{array}$} \\
\hline & Prolate & Oblate & Prolate & Oblate & & & \\
\hline 124 & 0.24 & -0.19 & 0.22 & -0.17 & 0.215 & 0.208 & $0.264(8)$ \\
\hline 126 & 0.19 & -0.18 & 0.18 & -0.15 & 0.186 & 0.170 & $0.1881(30)$ \\
\hline 128 & 0.16 & -0.16 & 0.15 & -0.12 & 0.160 & 0.143 & 0.1837 (49) \\
\hline 130 & 0.13 & -0.13 & 0.11 & -0.10 & 0.128 & -0.113 & $0.169(6)$ \\
\hline 132 & 0.11 & -0.10 & 0.07 & -0.10 & -0.070 & 0.000 & $0.1409(46)$ \\
\hline 134 & 0.05 & -0.05 & 0.01 & - & 0.000 & 0.000 & $0.120(10)$ \\
\hline 136 & 0.00 & - & 0.00 & - & -0.001 & 0.000 & 0.086 (19) \\
\hline 138 & 0.03 & - & 0.01 & - & -0.002 & 0.000 & 0.0309 (18) \\
\hline 140 & 0.15 & -0.10 & 0.12 & - & 0.104 & 0.116 & $0.1136(25)$ \\
\hline 142 & 0.17 & -0.12 & 0.16 & - & 0.141 & 0.145 & - \\
\hline
\end{tabular}




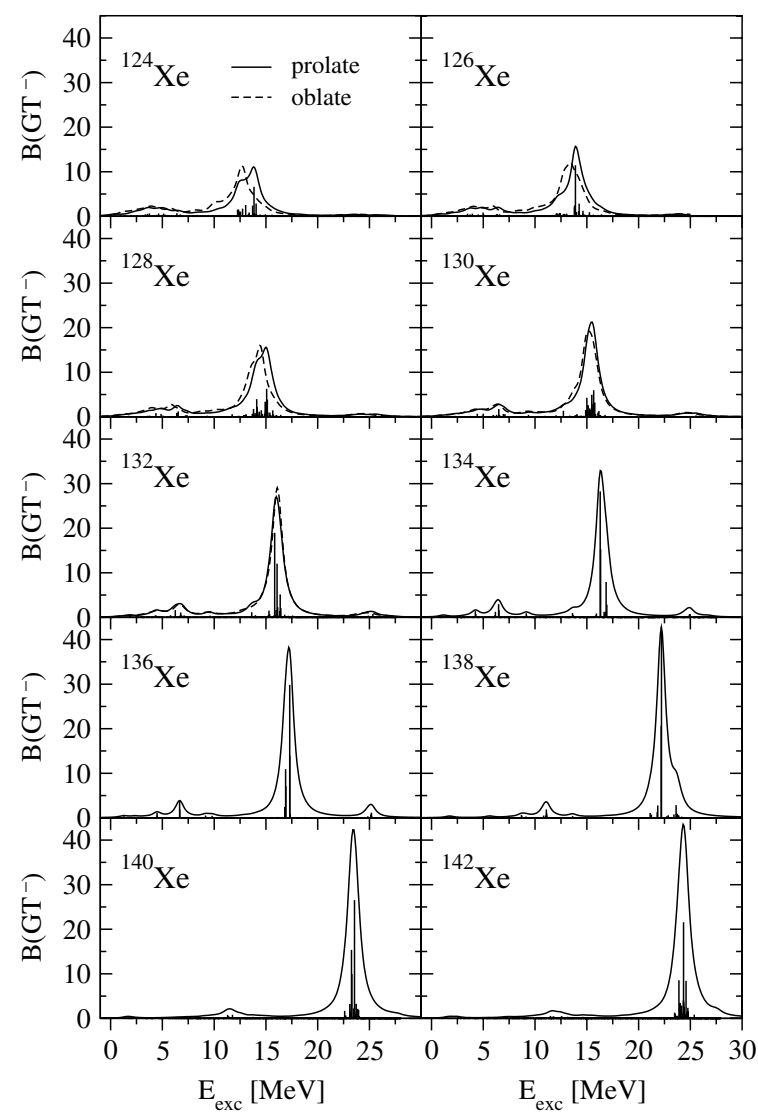

FIG. 2. Discrete and Gaussian-folded Gamow-Teller strength distributions $B\left(\mathrm{GT}^{-}\right)$for ${ }^{124-142} \mathrm{Xe}$, from a $\mathrm{HF}(\mathrm{Sk} 3)+\mathrm{BCS}(G)$ calculation. Two equilibrium deformations appear for ${ }^{124-132} \mathrm{Xe}$ : the solid line and the discrete spectrum, corresponding to the prolate shape, and the dashed line, corresponding to the oblate shape. The calculations for ${ }^{134,136,138} \mathrm{Xe}$ correspond to spherical shapes; those for ${ }^{140,142} \mathrm{Xe}$ correspond to prolate shapes (see Table II).

reactions obtained from them. The range of the excitation energy from 0 to $30 \mathrm{MeV}$ includes the resonance, which appears at around $13 \mathrm{MeV}$ in ${ }^{124} \mathrm{Xe}$ and moves slightly toward higher excitation energies as the mass number increases, reaching $25 \mathrm{MeV}$ in ${ }^{142} \mathrm{Xe}$.

Figure 3 shows the same calculations but for $\mathrm{GT}^{+}$transitions. As expected from the Ikeda sum rule, Eq. (2.9), the scale of the strengths is much smaller in this case. Table IV contains the summed $\mathrm{GT}^{ \pm}$strengths, their difference, the value of $3(N-Z)$, and the fulfillment of the Ikeda sum rule in percentage for the prolate shape of every isotope (which is generally the ground state), and for the oblate shape when there is a second minimum. The Ikeda sum rule is fulfilled up to a very high degree of accuracy in all cases.

The fragmentation observed in the $\mathrm{GT}^{+}$strength is reduced as the prolate energy minimum moves to the spherical region $\left({ }^{134,136,138} \mathrm{Xe}\right)$. A double-peak structure then becomes apparent, which was responsible for the larger width of these resonances in comparison with the ones in the $\mathrm{GT}^{-}$ distributions. A second peak with less strength, in the nearspherical shapes, appears $8 \mathrm{MeV}$ below the biggest one and moves accordingly with it as the mass number changes. It is

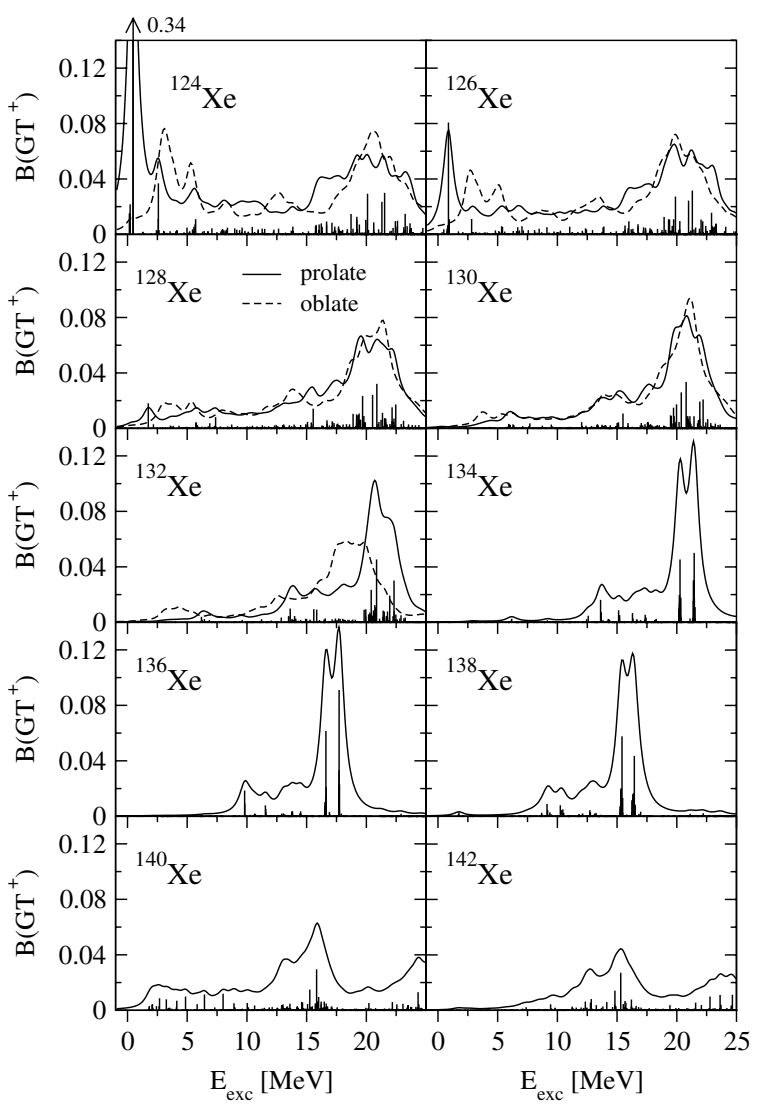

FIG. 3. The same as in Fig. 2 but for $\mathrm{GT}^{+}$strength distributions.

worth noticing the large single peak that appears at very low excitation energies in ${ }^{126} \mathrm{Xe}$ and also in ${ }^{124} \mathrm{Xe}$, reaching in the prolate shape of this last case a strength of $0.34 g_{A}^{2} /(4 \pi)$,

TABLE IV. Calculated summed $\mathrm{GT}^{-}$and $\mathrm{GT}^{+}$strengths [in units of $\left.g_{A}^{2} /(4 \pi)\right]$ for the ground state and the first $0^{+}$excited state (in brackets) of ${ }^{124-142} \mathrm{Xe}$, from a $\mathrm{HF}(\mathrm{Sk} 3)+\operatorname{BCS}(G)$ calculation. The difference between both summed strengths is compared with the value of $3(N-Z)$ to check the fulfillment of the Ikeda sum rule (in percentage).

\begin{tabular}{lccccc}
\hline \hline$A$ & $\sum \mathrm{GT}^{-}$ & $\sum \mathrm{GT}^{+}$ & $\sum \mathrm{GT}^{-}-\sum \mathrm{GT}^{+}$ & $3(N-Z)$ & $\%$ \\
\hline 124 & 48.62 & 1.15 & 47.47 & 48 & 98.90 \\
& $(48.34)$ & $(0.84)$ & $(47.50)$ & & $(98.96)$ \\
126 & 54.33 & 0.78 & 53.55 & 54 & 99.17 \\
& $(54.23)$ & $(0.71)$ & $(53.52)$ & & $(99.11)$ \\
128 & 60.16 & 0.60 & 59.56 & 60 & 99.26 \\
& $(60.18)$ & $(0.61)$ & $(59.57)$ & & $(99.27)$ \\
130 & 66.12 & 0.54 & 65.58 & 66 & 99.36 \\
& $(66.15)$ & $(0.56)$ & $(65.59)$ & & $(99.38)$ \\
132 & 72.12 & 0.51 & 71.61 & 72 & 99.46 \\
& $(71.83)$ & $(0.46)$ & $(71.37)$ & & $(99.13)$ \\
134 & 78.14 & 0.48 & 77.65 & 78 & 99.56 \\
136 & 83.59 & 0.47 & 83.12 & 84 & 98.95 \\
138 & 88.92 & 0.44 & 88.48 & 90 & 98.31 \\
140 & 94.69 & 0.65 & 94.04 & 96 & 97.96 \\
142 & 100.46 & 0.43 & 100.03 & 102 & 98.07 \\
\hline \hline
\end{tabular}


as indicated in the figure. This strength corresponds to a dominant GT transition from a $K^{\pi}=9 / 2^{+}$proton state to a $K^{\pi}=7 / 2^{+}$neutron state connecting the proton $g_{9 / 2}$ shell with the neutron $g_{7 / 2}$ shell. The occupation probability of the neutron state is small enough to allow the transition in ${ }^{124} \mathrm{Xe}$ and ${ }^{126} \mathrm{Xe}$, but when the number of neutrons increases, this state becomes blocked for GT transitions.

From the $\mathrm{GT}^{ \pm}$profiles of ${ }^{124-132} \mathrm{Xe}$ it is obvious that no clear distinction can be made between oblate and prolate deformations. It is only possible to distinguish these deformations in some cases when small energy windows are explored, as for example in the low- $B\left(\mathrm{GT}^{+}\right)$energy window of ${ }^{124,126} \mathrm{Xe}$. Similar studies on the effect of deformation in the GT strength distributions were made in the neutron-deficient $\mathrm{Hg}-\mathrm{Pb}-\mathrm{Po}$ region [31], and in the $A \simeq 70$ mass region [12].

The effect of deformation on the GT strength distributions can be observed more clearly in Fig. 4, where we compare spherical and deformed QRPA results. We show the examples of ${ }^{128} \mathrm{Xe}$, where two equilibrium shapes, oblate and prolate, are obtained, and ${ }^{140} \mathrm{Xe}$, where a prolate shape is predicted. In Fig. 4, plots upward correspond to deformed calculations with prolate shapes; plots downward correspond to spherical calculations. The left panels show the $\mathrm{GT}^{-}$strengths and the right ones the $\mathrm{GT}^{+}$strengths. As we can see in Fig. 4 the main effect of deformation is the stronger fragmentation of the strength, which is particularly clear on the $\mathrm{GT}^{+}$strength distributions because of the smaller scale. The positions of the peaks are also changed from spherical to deformed in a different way for each case.
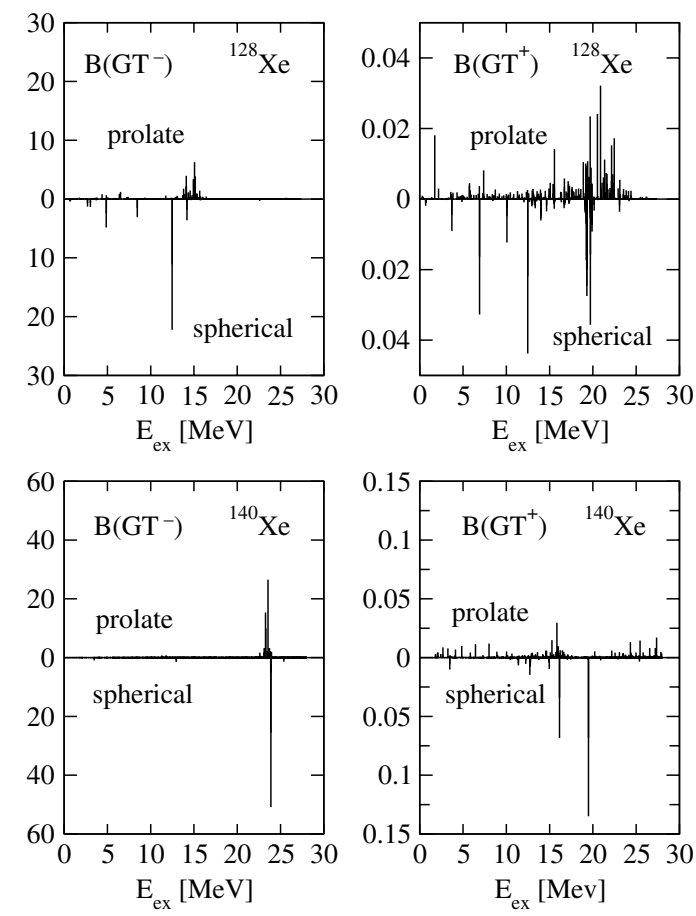

FIG. 4. GT strength distributions in ${ }^{128} \mathrm{Xe}$ (upper panels) and ${ }^{140} \mathrm{Xe}$ (lower panels). Left panels show $\mathrm{GT}^{-}$strengths; right panels show $\mathrm{GT}^{+}$strengths. Calculations with prolate and spherical shapes are plotted upward and downward, respectively.

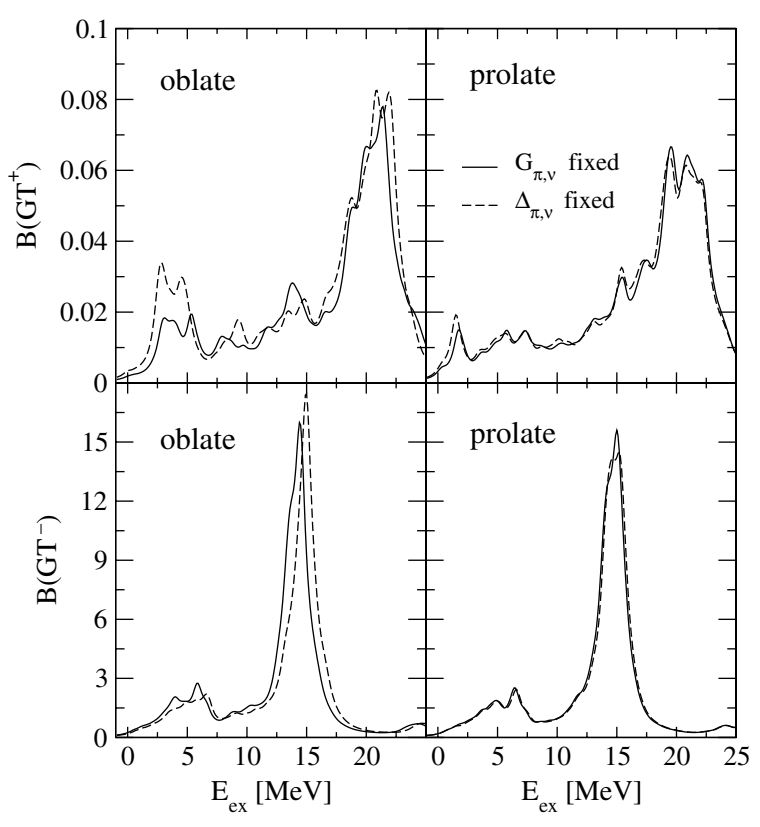

FIG. 5. Gaussian-folded Gamow-Teller strength distributions $B\left(\mathrm{GT}^{ \pm}\right)$in units of $g_{A}^{2} /(4 \pi)$ for both equilibrium shapes of ${ }^{128} \mathrm{Xe}$, from a $\mathrm{HF}(\mathrm{Sk} 3)+\mathrm{BCS}(G)$ calculation (solid line) and a $\operatorname{HF}(\operatorname{Sk} 3)+\operatorname{BCS}(\Delta)$ calculation (dashed line).

To compare the GT strength distributions obtained here with those coming from a $\mathrm{HF}(\mathrm{Sk} 3)+\operatorname{BCS}(\Delta)$ calculation of the single-particle levels, we show in Fig. 5 the distributions corresponding to both BCS pairing treatments (fixed gap and fixed strength) for the prolate and oblate equilibrium shapes of ${ }^{128} \mathrm{Xe}$. The results are very similar, as could be expected given the fact that the equilibrium nuclear shapes occur at very similar deformations and binding energies in both BCS treatments (see Fig. 1). In particular, for the prolate shape both distributions are almost identical, as expected because the values of the $G_{\pi, v}$ parameters were chosen to reproduce the $\Delta_{\pi, \nu}$ parameters precisely at this deformation. It is interesting to note that also at the oblate minimum these pairing strengths nearly reproduce the values of the phenomenological pairing gaps, since as seen in Fig. 1 they are very close to the ones at the prolate minimum. Therefore the distributions for the oblate case are also very similar for the two pairing treatments. In the case of spherical equilibrium shapes, as for example ${ }^{136} \mathrm{Xe}$, the GT strength distributions from fixed-pairing-gap and fixed-pairing-strength calculations are indistinguishable.

\section{SUMMARY AND CONCLUSIONS}

The Xe isotopes are of considerable theoretical interest because they participate in a variety of double- $\beta$ decay processes and because they belong to a nuclear shape transitional region. In addition, from the experimental point of view, the stable Xe isotopes have been used as moving targets in charge-exchange reactions to test new facilities, where the unstable Xe isotopes will be explored in the near future. The present work has addressed these topics by predicting stable nuclear shapes and GT strength distributions, which are a 
fundamental tool to calculate single- and double- $\beta$ transition matrix elements and half-lives, as well as cross sections of charge-exchange reactions under the appropriate kinematic conditions.

For even-even Xe isotopes with mass numbers from 124 to 142 , we have studied the GT strength distributions using a deformed $p n$ QRPA formalism with ph and pp spin-isospin separable residual interactions. The quasiparticle mean field is obtained from an axially deformed HF approach, with the Skyrme interaction $\mathrm{Sk} 3$, including pairing correlations between like nucleons in the BCS approximation by using either fixed gaps $\left(\Delta_{\pi, v}\right)$ or fixed pairing interaction strength $\left(G_{\pi, \nu}\right)$. The HF + BCS mean field has been also used to consistently determine the ph coupling constant for every isotope, whose average value has been finally used for all of them. The pp coupling constant has been fixed to approximately reproduce the half-life of the three $\beta^{-}$-unstable $\mathrm{Xe}$ isotopes included in this work.

From the energy-deformation curves, an oblate-prolate shape coexistence is predicted in ${ }^{124-132} \mathrm{Xe}$ with a low-energy barrier between them. The isotopes ${ }^{134-138} \mathrm{Xe}$ are predicted to be spherical, whereas the prolate shape seems to be strongly favored in ${ }^{140-142} \mathrm{Xe}$. In general, a fixed-pairing-strength calculation increases the binding energy of the spherical shape region. The deformation dependence of the pairing energy gaps from a fixed-pairing-strength calculation has been also shown. The self-consistent quadrupole deformations of the ground state derived within the $\mathrm{HF}+\mathrm{BCS}$ procedure are in agreement with independent theoretical calculations as well as with the experimental upper bounds extracted from $B(E 2)$ transitions in the whole chain of $\mathrm{Xe}$ isotopes under study.

The $\mathrm{GT}^{-}$strength distributions for the equilibrium shapes are dominated by a single peak moving to higher excitation energies and gathering more strength as the number of neutrons increases, as expected. In the cases where two equilibrium shapes are predicted, there is no strong dependence of the GT strength distribution on the equilibrium shape, at least when a wide range of excitation energy is considered.

In the case of the $\mathrm{GT}^{+}$transitions, the strength is more fragmented, giving rise to a richer structure in the energy distribution. This fragmentation decreases as the energy minima move to the spherical region, where a double-peaked resonance appears, as observed in ${ }^{134-138} \mathrm{Xe}$. The lightest isotopes, ${ }^{124} \mathrm{Xe}$ and ${ }^{126} \mathrm{Xe}$, show a very high peak from a single transition at very low excitation energies, but this transition is blocked in the isotopes with more neutrons. With such a complex structure, the influence of the sign of the nuclear deformation (oblate or prolate) in ${ }^{124-132} \mathrm{Xe}$ is more apparent on the $\mathrm{GT}^{+}$strength distributions than on the $\mathrm{GT}^{-}$transitions, but in any case it does not seem to be critical. However, the GT strength distributions obtained from spherical or deformed shapes show different features related to the energy location of the main peaks and to the fragmentation of the strength. From the accumulated $\mathrm{GT}^{ \pm}$strengths up to $30 \mathrm{MeV}$ of excitation energy, it has been shown that the Ikeda sum rule is fulfilled up to a very high percentage (see Table IV).

Theoretical studies of GT strengths and related observables such as, in particular, charge-exchange reaction cross sections are necessary to help in the event simulation work of these kind of processes, many of which will be measured at the new FAIR-GSI facility. Theoretical work in this direction is in progress.

\section{ACKNOWLEDGMENTS}

This work was supported by the Ministerio de Educación y Ciencia (Spain) under Contract Nos. FIS2005-00640 and BFM2003-04147-C02-01. O.M. thanks the Ministerio de Educación y Ciencia (Spain) for financial support. R.A.R. thanks the I3P Programme (CSIC, Spain) for financial support. We also acknowledge participation in the European Collaborations EURONS (RII3-506065), ILIAS (RII3-CT-2004-506222), and INTAS-03-54-6545.
[1] EXL Technical Report (2005) and private communication.

[2] C. D. Goodman, C. A. Goulding, M. B. Greenfield, J. Rapaport, D. E. Bainum, C. C. Foster, W. G. Love, and F. Petrovich, Phys. Rev. Lett. 44, 1755 (1980); T. N. Taddeucci, C. A. Goulding, T. A. Carey, R. C. Byrd, C. D Goodman, C. Gaarde, J. Larsen, D. Horen, J. Rapaport, and E. Sugarbaker, Nucl. Phys. A469, 125 (1987); F. Osterfeld, Rev. Mod. Phys. 64, 491 (1992).

[3] H. Ejiri, Phys. Rep. 338, 265 (2000).

[4] A. Faessler and F. Simkovic, J. Phys. G 24, 2139 (1998); J. Suhonen and O. Civitarese, Phys. Rep. 300, 123 (1998); S. R. Elliott and P. Vogel, Annu. Rev. Nucl. Part. Sci. 52, 115 (2002); J. D. Vergados, Phys. Rep. 361, 1 (2002); H. V. Klapdor-Kleingrothaus, Sixty Years of Double Beta Decay (World Scientific, Singapore, 2001).

[5] V. I. Tretyak and Y. G. Zdesenko, At. Data Nucl. Data Tables 80, 83 (2002).

[6] A. S. Barabash, Czech. J. Phys. 56, 437 (2006).

[7] G. Audi, O. Bersillon, J. Blachot, and A. H. Wapstra, Nucl. Phys. A729, 3 (2003).
[8] R. Alvarez-Rodriguez, P. Sarriguren, E. Moya de Guerra, L. Pacearescu, A. Faessler, and F. Šimkovic, Phys. Rev. C 70, 064309 (2004).

[9] R. Wyss et al., Nucl. Phys. A505, 337 (1989); N. Yoshinaga and K. Higashiyama, Phys. Rev. C 69, 054309 (2004).

[10] W. Lieberz, A. Dewald, W. Frank, A. Gelberg, W. Krips, D. Lieberz, R. Wirowski, and P. von Brentano, Phys. Lett. B240, 38 (1990).

[11] X. W. Pan, J. L. Ping, D. H. Feng, J. Q. Chen, C. L Wu, and M. W. Guidry, Phys. Rev. C 53, 715 (1996).

[12] P. Sarriguren, E. Moya de Guerra, A. Escuderos, and A. C. Carrizo, Nucl. Phys. A635, 55 (1998); P. Sarriguren, E. Moya de Guerra, and A. Escuderos, ibid. A658, 13 (1999); A691, 631 (2001); Phys. Rev. C 64, 064306 (2001).

[13] E. Poirier et al., Phys. Rev. C 69, 034307 (2004); E. Nacher et al., Phys. Rev. Lett. 92, 232501 (2004).

[14] M. Beiner, H. Flocard, N. Van Giai, and P. Quentin, Nucl. Phys. A238, 29 (1975).

[15] H. Flocard, P. Quentin, A. K. Kerman, and D. Vautherin, Nucl. Phys. A203, 433 (1973). 
[16] J. Krumlinde and P. Moeller, Nucl. Phys. A417, 419 (1984); P. Moeller and J. Randrup, ibid. A514, 1 (1990).

[17] M. Hirsch, A. Staudt, K. Muto, and H. V. KlapdorKleingrothaus, Nucl. Phys. A535, 62 (1991).

[18] H. Homma, E. Bender, M. Hirsch, K. Muto, H. V. KlapdorKleingrothaus, and T. Oda, Phys. Rev. C 54, 2972 (1996).

[19] K. Muto, E. Bender, T. Oda, and H. V. Klapdor-Kleingrothaus, Z. Phys. A 341, 407 (1992).

[20] J. Engel, P. Vogel, and M. R. Zirnbauer, Phys. Rev. C 37, 731 (1988); V. A. Kuz'min and V. G. Soloviev, Nucl. Phys. A486, 118 (1988).

[21] A. Bohr and B. Mottelson, Nuclear Structure (Benjamin, New York, 1975).

[22] E. Moya de Guerra, Phys. Rep. 138, 293 (1986).

[23] D. Vautherin and D. M. Brink, Phys. Rev. C 5, 626 (1972); D. Vautherin, ibid. 7, 296 (1973); J. Negele and D. Vautherin, ibid. 5, 1472 (1972); 11, 1031 (1975); P. Quentin and H. Flocard, Annu. Rev. Nucl. Part. Sci. 28, 523 (1978); J. Friedrich and P.-G. Reinhard, Phys. Rev. C 33, 335 (1986); E. Chabanat, P. Bonche, P. Haensel, J. Meyer, and R. Schaeffer, Nucl. Phys. A627, 710 (1997); A635, 231 (1998).
[24] G. F. Bertsch and H. Esbensen, Ann. Phys. (NY) 209, 327 (1991); E. Garrido, P. Sarriguren, E. Moya de Guerra, and P. Schuck, Phys. Rev. C 60, 064312 (1999).

[25] P. Sarriguren, E. Moya de Guerra, and R. Nojarov, Phys. Rev. C 54, 690 (1996).

[26] G. F. Bertsch and S. F. Tsai, Phys. Rep. 18, 127 (1975); S. Krewald, V. Klemt, J. Speth, and A. Faessler, Nucl. Phys. A281, 166 (1977).

[27] I. Hamamoto and H. Sagawa, Phys. Rev. C 60, 064314 (1999); M. Bender, J. Dobaczewski, J. Engel, and W. Nazarewicz, ibid. 65, 054322 (2002); N. Van Giai, C. Stoyanov, and V. V. Voronov, ibid. 57, 1204 (1998).

[28] G. A. Lalazissis, S. Raman, and P. Ring, At. Data Nucl. Data Tables 71, 1 (1999).

[29] P. Moeller, J. R. Nix, W. D. Myers, and W. J. Swiatecki, At. Data Nucl. Data Tables 59, 185 (1995).

[30] S. Raman, C. H. Malarkey, W. T. Milber, C. W. Nestor Jr., and P. H. Stelson, At. Data Nucl. Data Tables 36, 1 (1987).

[31] O. Moreno, P. Sarriguren, R. Alvarez-Rodriguez, and E. Moya de Guerra, Phys. Rev. C 73, 054302 (2006). 